\title{
Systemic Evaluation on Antifungal Susceptibility of Keratitis Associated Fungal Pathogens in Eastern India
}

Suman Saha ${ }^{{ }^{*}}$, Jayangshu Sengupta ${ }^{1}$, Debdulal Banerjee ${ }^{2}$, Sunayana Saha ${ }^{3}$, Archana Khetan $^{1,4}$ and Santi M. Mandal ${ }^{2}$,

${ }^{1}$ Priyamvada Birla Aravind Eye Hospital, Loudon Street, Kolkata, India

${ }^{2}$ Dept of Botany and Forestry, Vidyasagar University, Midnapore, West Bengal, India

${ }^{3}$ Dept of Biological Science, Presidency University, Kolkata, India

${ }^{4}$ Central Research Facility, Indian Institute of Technology Kharagpur, Kharagpur, West Bengal, India

*Corresponding author: Suman Saha, Priyamvada Birla Aravind Eye Hospital, 10, Loudon Street, Kolkata, India, Tel: 9133228943, E-mail: sumansaha13@hotmail.com

Rec date: Jan 10, 2014, Acc date: May 01, 2014, Pub date: May 03, 2014

Copyright: ( 2014 Saha S, et al. This is an open-access article distributed under the terms of the Creative Commons Attribution License, which permits unrestricted use, distribution, and reproduction in any medium, provided the original author and source are credited.

\begin{abstract}
Assessment of the keratitis associated fungal pathogens and realizes their antifungal sensitivity pattern in Eastern India for better guidance and appropriate choice of antifungal drugs was made. A retrospective, non-comparative study was conducted on 248 fungal keratitis patients between August'2009-July'2012. Corneal scraping was performed and evaluated antifungal susceptibility in all cases. Culture positive fungal keratitis were found in $77.82 \%$ cases in which the most predominating pathogen was Aspergillus $s p$ of $46.63 \%$ including Aspergillus fumigatus in 41 cases (45.55\%) and Aspergillus flavus in 25 cases (27.77\%). Aspergillus $s p$ was mostly sensitive to voriconazole next to natamycin and amphotericin B. The second most common pathogen was Candida albicans in 36 cases $(18.94 \%)$. Candida $\mathrm{sp}$ was mostly sensitive to amphotericin B followed by natamycin. Candida $s p$ was relatively insensitive to fluconazole and voriconazole. The third common fungal pathogen was Fusarium $s p$ in 30 cases, among them $F$. solani in 27 cases $(90 \%)$. Fusarium $s p$ was mostly sensitive to voriconazole next to natamycin. Aspergillus, Candida and Fusarium were insensitive to fluconazole, micronazole and itraconazole. Aspergillus $s p$ is the most common pathogen of fungal keratitis followed by Candida and Fusarium sp. Voriconazole is still the first choice in the treatment of mould keratitis. Early diagnosis and treatments are vital for better management of fungal keratitis.
\end{abstract}

Keywords: Fungal keratitis; Antifungal sensitivity; Voriconazole

\section{Introduction}

Ocular fungal keratitis is one of the most common infectious ocular diseases in India [1]. According to the World Health Organization report, it was estimated that ocular trauma and corneal ulceration resulting 1.5 to 2 million new patients annually posing a major health problem in developing country [2]. Due to shortage and unavailability of efficient and broad spectrum antifungal agents the therapeutic effects with antifungal drugs are not found to be effective. There are so many fungal species that have been reported as pathogens of fungal keratitis, and the antifungal susceptibility of the different species are different. In this study we retrospectively analyzed the pathogens of fungal keratitis in the cornea services of a tertiary eye care hospital in Kolkata, India from August 2009 to July 2012 and the susceptibility of common species to seven antifungal agents hoping to find out the appropriate administration of antifungal drugs in the treatment of fungal keratitis.

\section{Methods}

After obtaining approval from the institutional review board, the medical records of 471 clinically diagnosed patients of microbial keratitis, who attained Cornea service of Priyamvada Birla Aravind Eye Hospital, a tertiary eye care hospital in Kolkata, India, from August 2009 to July 2012, following the introduction of in-house microbiology services, were evaluated retrospectively. The evaluation were based on duration of symptoms, predisposing factors like trauma, associated ocular conditions, any systemic diseases, therapy received before presentation, history of corticosteroids use and previous eye surgery. The Cornea service protocol for management of patients with a clinical diagnosis of microbial keratitis was identical for all patients in this study. Corneal scraping was performed in all patients by the ophthalmologist using a sterile 15 Bard Parker surgical blade, following the instillation of local anesthetic eye drop such as $0.5 \%$ proparacaine, under aseptic conditions. The material was obtained from the active margin and base of the ulcer following debridement of superficial mucus. In patients with deep corneal infiltrates with or without anterior chamber exudates, a deep scraping, corneal biopsy or anterior chamber tap was additionally performed. In patients where therapeutic keratoplasty was performed, corneal scraping was repeated at the time of surgery, and excised corneal buttons were subjected to microbiological and histopathological procedures.

The material obtained was directly smeared on a labelled slide for $10 \% \mathrm{KOH}$ wet mount, Gram's staining [3] and incubated directly in solid media (10\% sheep blood agar, Sabourand's Dextrose Agar (SDA), Potato Dextrose Agar (PDA), nutrient agar and chocolate agar) in a row of C-shaped streaks. Brain heart infusion broth was used for a few patients in whom fungal keratitis was strongly suspected. The culture plates (10\% sheep blood agar plate, chocolate agar and brain heart infusion broth) were kept for 10 days at $37^{\circ} \mathrm{C}$, and discarded if no growth was obtained and no turbidity was seen after 10 days. The SDA 
Citation: Saha S, Sengupta J, Banerjee D, Khetan A and Mandal M (2014) Systemic Evaluation on Antifungal Susceptibility of Keratitis Associated Fungal Pathogens in Eastern India. J Med Microb Diagn 3: 134. doi:10.4172/2161-0703.1000134

Page 2 of 5

and PDA plates were observed for 21 days in room temperature $\left(25-27^{\circ} \mathrm{C}\right)$ in aerobic condition. Any growth obtained was further identified by macroscopic and microscopic aspects of texture, pigmentation, mycelium arrangement and conidium types by lactophenol cotton blue mount. Yeast was differentiated by germ tube formation as well as using biochemical test kit (Hi-Media, India) and sporulation was observed on Czapek Dox medium. The culture was considered positive if the growth of the same fungal species was found in more than one solid media.

Patients with a diagnosis of fungal keratitis as determined by positive microbiology result (patients fungal elements identified in $10 \%$ potassium hydroxide $(\mathrm{KOH})$ wet mount preparation and gram staining, and positive growth for same fungus in at least two solid media) were identified. Antifungal drug sensitivity tests were performed in accordance with national committee for clinical laboratory standards (NCCLS) guidelines. The control strain was Candida albicans (ATCC 10231). In brief seven kind of antifungal disc (Hi Media, India) were used which include voriconazole, natamycin, amphotericin B, fluconazole, micronazole, ketoconazole and itraconazole. The selective stains (Aspergillus fumigatus, Aspergillus flavus, Fusarium solani, and Candida albicans) to be tested were inoculated into SDA media, cultured for 2-5 days and the inoculum were diluted to $0.5 \times 10^{4} \mathrm{CFU} / \mathrm{ml}$ with saline water. The inoculum $(0.5$ $\mathrm{ml}$ ) were plated in SDA media, dried for $15 \mathrm{~min}$ at $37^{\circ} \mathrm{C}$ then antifungal discs were placed on the media surface by following disc diffusion methods. The plates were incubated at $30^{\circ} \mathrm{C}$ and the zone diameters were observed after $48 \mathrm{hrs}$ incubation. The zone diameters were measured at which colonies were remaining normal. The zone diameters were interpreted with the guideline proposed by the manufacturer's guidelines. E-test was performed for selective antifungal (voriconazole, Amphotericin B, ketoconazole, itraconazole) (Hi Media, India) to find out the minimum inhibitory concentration after $48 \mathrm{hrs}$ incubation at $30^{\circ} \mathrm{C}$.

\section{Results}

Two hundred forty three patients were diagnosed as fungal keratitis based on clinical findings as well as observation of fungal filaments in $\mathrm{KOH}$ mount and Grams staining. Of these 190 patients (76.61\%) were culture positive fungal keratitis and remaining 58 patients were only smearing positive. There were more male patients (132/190 i.e. $69.47 \%$ ) compared to female patients (58/190 i.e. $30.53 \%)$. The mean patient age was 56 years. There was a history of trauma in 101 patients (101/190 i.e. $53.15 \%)$ followed by history of steroid usage in 31 patients (31/190 i.e. $16.31 \%)$, chronic eye diseases in 20 patients (20/190 i.e. $10.52 \%)$ and 15 patients had diabetes history. No obvious predisposing factor could be identified in remaining patients. Maximum culture positivity was found from corneal scraping, 132 of $190(132 / 190$ i.e. $69.47 \%)$ followed by corneal button obtained during therapeutic keratoplasty 33 of 190 (33/190 i.e. 17.36\%) anterior chamber tap 20 of 190 (20/190 i.e. 10.52\%) and deep corneal biopsy in 5 patients (5/190 i.e. $2.63 \%$ ). The predominating isolate was genus Aspergillus in 90 patient (46.63\%) of which Aspergillus fumigatus was the most common species in 41 patients (45.55\%) followed by Aspergillus flavus in 25 patients (27.77\%). The second most common pathogen was genus Candida in 36 cases (18.94\%) with Candida albicans in 35 cases along with one unidentified Candida sp. The third common fungal pathogen was Fusarium in 30 cases (15.78\%) with Fusarium solani in 27 cases (90\%) and Fusarium oxysporium in 3 cases $(10 \%)$ (Table 1$)$.

\begin{tabular}{|c|c|c|}
\hline Fungal Pathogens Isolated & Number & Percentage (\%) \\
\hline Aspergillus genus & 90 & $46.63 \%$ \\
\hline Aspergillus fumigatus & 41 & \\
\hline Aspergillus flavus & 25 & \\
\hline Aspergillus terrus & 09 & \\
\hline Aspergillus tamari & 06 & \\
\hline Aspergillus niger & 04 & \\
\hline Aspergillus nidulans & 02 & \\
\hline Aspergillus other species & 03 & \\
\hline Candida genus & 36 & $18.94 \%$ \\
\hline Candida albicans & 35 & \\
\hline Candida other species & 01 & \\
\hline Fusarium genus & 30 & $15.78 \%$ \\
\hline Fusarium solani & 27 & \\
\hline Fusarium oxysporium & 03 & \\
\hline Penicillium sp & 11 & \\
\hline Scedosporium $s p$ & 03 & \\
\hline
\end{tabular}


Citation: Saha S, Sengupta J, Banerjee D, Khetan A and Mandal M (2014) Systemic Evaluation on Antifungal Susceptibility of Keratitis Associated Fungal Pathogens in Eastern India. J Med Microb Diagn 3: 134. doi:10.4172/2161-0703.1000134

Page 3 of 5

\begin{tabular}{|l|l|l|}
\hline Rhizopus $s p$ & 02 & \\
\hline Bipolaris $s p$ & 02 & \\
\hline Cladosporium $s p$ & 02 & \\
\hline Alternaria $s p$ & 02 & \\
\hline Curvularia $s p$ & 02 & \\
\hline Unidentified sp & 09 & \\
\hline Total & 190 & \\
\hline
\end{tabular}

Table 1: Distribution of fungal species in fungal keratitis in Eastern India Aug 2009-July 2012

Ninety two $(48.42 \%)$ patients with culture positive fungal keratitis patients showed response to topical medical therapy with the formation of corneal scar. The remaining 98 patients $(51.58 \%)$ of mycotic keratitis patients needed therapeutic keratoplasty. This included 39 cases of 90 Aspergillus keratitis (43.33\%) and 14 cases of $23(60.86 \%)$ of Fusarium keratitis. In our study all patients with Candida $s p$ infections required a surgical intervention. 27 patients among $190(14.21 \%)$ culture positive fungal keratitis cases need immediate therapeutic keratoplasty at presentation. Voriconazole was most effective antifungal against all common pathogens isolated from fungal keratitis except Candida $s p$. Fluconazole was resistant against all common species. Natamycin and Amphotericin B shows inhibition zone against all isolated specie0073 (Table 2). Voriconazole shows lowest MIC against Aspergillus and Fusarium $s p$ followed by amphotericin B. Ketoconazole and Itraconazole showed lowest MIC against Candida sp (Table 3).

\begin{tabular}{|c|c|c|c|c|}
\hline \multirow{2}{*}{$\begin{array}{l}\text { Antifungals } \\
7 \text { Discs }\end{array}$} & \multicolumn{4}{|c|}{ Selective most common fungal species } \\
\hline & $\begin{array}{l}\text { A.fumigatus } \\
n=10\end{array}$ & $\begin{array}{l}\text { A.flavus } \\
\mathrm{n}=07\end{array}$ & $\begin{array}{l}\text { Candida albicans } \\
\mathrm{n}=5\end{array}$ & $\begin{array}{l}\text { F. solani } \\
\mathrm{n}=03\end{array}$ \\
\hline \multicolumn{5}{|c|}{ Amphotericin B } \\
\hline $\mathrm{s}$ & $10(100 \%)$ & $07(100 \%)$ & $05(100 \%)$ & $03(100 \%)$ \\
\hline $\mathrm{R}$ & 00 & 00 & 00 & 00 \\
\hline \multicolumn{5}{|l|}{ Voriconazole } \\
\hline $\mathrm{s}$ & $10(100 \%)$ & $07(100 \%)$ & $01(20 \%)$ & $02(66.67 \%)$ \\
\hline $\mathrm{R}$ & 00 & 00 & $04(80 \% 0$ & $01(33.33 \%)$ \\
\hline \multicolumn{5}{|c|}{ Ketoconazole } \\
\hline S & 00 & $01(14.28 \%)$ & $04(80 \%)$ & 00 \\
\hline $\mathrm{R}$ & $10(100 \%)$ & $06(85.72 \%)$ & $01(20 \%)$ & $03(100 \%)$ \\
\hline \multicolumn{5}{|l|}{ Fluconazole } \\
\hline S & 00 & 00 & 00 & 00 \\
\hline $\mathrm{R}$ & $10(100 \%)$ & $07(100 \%)$ & $05(100 \%)$ & $03(100 \%)$ \\
\hline \multicolumn{5}{|l|}{ Miconazole } \\
\hline $\mathrm{s}$ & $02(20 \%)$ & $02(28.57 \%)$ & 00 & $02(66.67 \%)$ \\
\hline $\mathrm{R}$ & $08(80 \%)$ & 05 (71.43\%) & $05(100 \%)$ & $01(33.33 \%)$ \\
\hline \multicolumn{5}{|l|}{ Itraconazole } \\
\hline $\mathrm{s}$ & $06(60 \%)$ & $02(28.57 \%)$ & $04(80 \%)$ & $02(66.67 \%)$ \\
\hline $\mathrm{R}$ & $04(40 \%)$ & $05(71.43 \%)$ & $01(20 \%)$ & $01(33.33 \%)$ \\
\hline Natamycin & & & & \\
\hline
\end{tabular}




\begin{tabular}{|l|l|l|l|l|}
\hline$S$ & $10(100 \%)$ & $07(100 \%)$ & $05(100 \%)$ & $03(100 \%)$ \\
\hline$R$ & 00 & 00 & 00 & 00 \\
\hline S= Susceptible, R= Resistant.
\end{tabular}

Table 2: Antifungal Suseptibility of 25 strains in Eastern India during Aug 2009-July 2012

\begin{tabular}{|l|l|l|l|l|}
\hline \multirow{2}{*}{$\begin{array}{l}\text { Antifungals } \\
\text { MIC Strips }\end{array}$} & \multicolumn{4}{|l|}{ Selective most common fungal species } \\
\cline { 2 - 5 } & $\begin{array}{l}\text { A.fumigatus } \\
\mathrm{n}=10\end{array}$ & $\begin{array}{l}\text { A.flavus } \\
\mathrm{n}=07\end{array}$ & $\begin{array}{l}\text { Candida albicans } \\
\mathrm{n}=5\end{array}$ & $\begin{array}{l}\text { F. solani } \\
\mathrm{n}=03\end{array}$ \\
\hline Amphotericin B & $0.128-0.064$ & $0.128-0.064$ & $0.64-1.2$ & 1 \\
\hline Voriconazole & $0.06-0.012$ & $0.128-0.06$ & 4 & 2 \\
\hline Ketoconazole & 4 & 4 & $0.032-0.016$ & 8 \\
\hline Itraconazole & 1 & $0.5-0.25$ & $0.25-0.128$ & 4 \\
\hline
\end{tabular}

Table 3: Minimum inhibitory Concentration $(\mu \mathrm{g} / \mathrm{ml})$ of 4 antifungal drugs against 25 strains in Eastern India during Aug 2009-July 2012

\section{Discussion}

Aspergillus species (55.4\%) and Candida species (18.91\%) were found to be the major etiologic agents of fungal keratitis in this study followed by Fusarium $s p(10.81 \%)$. Other studies have implicated Fusarium species (37-62\%) and Aspergillus species (24-30\%) as major pathogens with Dematiaceous fungi as the cause of $8-16.7 \%$ of patients with fungal keratitis [4]. In India, Aspergillus $s p$ is the main etiological organism responsible for mycotic keratitis followed by Fusarium $s p$ except in South India where Fusarium keratitis was maximum up to 43\%. In Nepal, Upadhyay et al. [5] found that Aspergillus sp. accounted for $47 \%$ of all fungal pathogens followed by Candida $s p$ (13.2\%) and Fusarium $s p$ (11.7\%). Gopinathan et al. [6] from India have reported Candida $s p$ as a rare fungal corneal pathogen $(0.7 \%)$. In another series of 24 patients from Wills Eye Hospital, Philadelphia, Candida $s p$ was identified in $45.8 \%$ of patients of fungal keratitis [7]. The relatively high isolation of Candida $s p$ in this set of patients deserves attention. It has been reported that Candida infection is more common in temperate climate of the West compared to the tropical climate of East. However, it is also known that the growth rate of yeast is maximum in warm and moist condition [8]. Basak et al. [9] reported $1.1 \%$ incidence of Candida positive patients among 509 mycotic keratitis patients. This study was conducted in a hospital with a rural population base. Factors other than climatic conditions, such as altered local defense mechanisms and immune suppression may also be responsible for the higher incidence of Candida infection in some studies compared to others.

Surgical intervention in the form of therapeutic keratoplasty continues to be an important mode of management. In this study $48.42 \%$ patients showed response to medical therapy while $51.58 \%$ of mycotic keratitis patients including $43.33 \%$ Aspergillus keratitis and $60.86 \%$ of Fusarium keratitis needed therapeutic keratoplasty. In contrast with Vamuganti et al. [10] report that maximum fungal species identified from corneal buttons after therapeutic keratoplasty were Fusarium $s p$ in $39 \%$ and Aspergillus $s p$ in 33\%. Surprisingly, all Candida keratitis of this series needed surgical intervention. Regina et al. [11] reported from Texas that out of 29 Candida Keratitis patients,
15 patient's required surgical intervention of which 13 patient had therapeutic penetrating keratoplasty and 2 eyes needed enucleation.

Antifungal sensitivity test has developed greatly in the recent years, various antifungal drug sensitivity methods such as agar dilution, disc diffusion, concentration gradient and liquid medium dilution are applied in various laboratories in India. We used disc diffusion methods by following NCCLS guidelines (2002) where the test mediators, inoculum concentration, incubation temperature for different mycelial fungus are clearly defined Genus Aspergillus, Candida, and Fusarium were the most common fungal pathogen in this study of which the common species were Aspergillus fumigatus, Aspergillus flavus, Candida albicans, Fusarium solani and Fusarium oxysporium. The sensitivities of these species to antifungal drugs were tested and analysed. Commonly used antifungal medicines for sensitivity test were polyene (natamycin, amphotericin B) and azoles (Voriconazole, Ketoconazole, Miconazole, Fluconazole, Itraconazole), among them depending upon the severity of the ulcer topical natamycin and voriconazole was used as a first line antifungal treatment in fungal keratitis. This study showed both Aspergillus and Fusarium were more sensitive to voriconazole then natamycin and Amphotericin B was effective against Candida. But in India it is very difficult to widely use topical voriconazole (2\%) due to its high price. Amphotericin B was quite sensitive to genus Aspergillus and Fusarium but due to poor penetration in cornea it was not used in such keratitis. It also requires high dosage to ensure the adequate concentration in eye [12]. Natamycin is the standard of care in many countries especially in developing countries where natamycin was effective in superficial infection not in severe case [13]. As we are sitting in the heart of the city so we are getting maximum number of severe patients from rural population. Then voriconazole was choosen as a topical medication therapy as it showed maximum susceptibility against filamentous fungi with lowest MIC correlating with the study of Lalitha et al. [2] reported that voriconazole had a lowest MIC against Aspergillus as Fluconazole and miconazole showed resistance against maximum number of species. Ketoconazole and itaconazole showed invitro sensitivity against Candida rather than other filamentary fungi. However, as showed in this study that resistance of Fusarium and Aspergillus to azoles had increased greatly. The new antifungal 
Citation: Saha S, Sengupta J, Banerjee D, Khetan A and Mandal M (2014) Systemic Evaluation on Antifungal Susceptibility of Keratitis Associated Fungal Pathogens in Eastern India. J Med Microb Diagn 3: 134. doi:10.4172/2161-0703.1000134

Page 5 of 5

voriconazole has proved as a high efficient broad spectrum and low toxicity medication for the treatment of filamentous fungi keratitis. Other new antifungals such as posaconazole and caspofungin have been developed in recent years [14], but the results were different because of the different fungal spectrum in different country and location. It is important to remember that all the results of antifungal sensitivity tests in vitro are completely not coincident with the therapeutic effects in vivo due to immune defense of hosts also plays important roles in the occurrence of fungal infection. If the pathogen was sensitive to an antifungal treatment with this medication will be effective up to $90 \%$ of the patients and in case of resistance therapy it will be effective in $60 \%$ of patients [15]. The disease takes a turn for improvement after administration of antifungal agents which promote the ulcers to heel gradually. So it is proved that early diagnosis and timely intervention with antifungal drugs are vital to good therapeutic effects in fungal keratitis.

\section{Conclusion}

Our study addresses that Aspergillus sp. remains the most predominant organism and Candida $s p$. were isolated in high numbers. Topical therapy is not always sufficient to eradicate infection with fungal keratitis irrespective of the identified organism through Aspergillus and Fusarium keratitis seem to show some response to medical management. Therapeutic keratoplasty continues to remain an important treatment modality particularly in infection with Candida sp. Voriconazole is the first choice in the treatment of filamentous fungal keratitis followed by natamycin and amphotericin B. Early diagnosis and timely antifungal treatments are very important for therapeutically effective in fungal keratitis management.

\section{References}

1. Thomas PA (2003) Fungal infections of the cornea. Eye (Lond) 17: 852-862.

2. Lalitha P, Shapiro BL, Srinivasan M, Prajna NV, Acharya NR, et al. (2007) Antimicrobial susceptibility of Fusarium, Aspergillus, and other filamentous fungi isolated from keratitis. Arch Ophthalmol 125: 789-793.
3. Bharathi MJ, Ramakrishnan R, Vasu S, Meenakshi R, Palaniappan R (2003) Epidemiological characteristics and laboratory diagnosis of fungal keratitis. A three-year study. Indian J Ophthalmol 51: 315-321.

4. Anderson KL, Mitra S, Salouti R, Pham TA, Taylor HR (2004) Fungal keratitis caused by Paecilomyces lilacinus associated with a retained intracorneal hair. Cornea 23: 516-521.

5. Upadhyay MP, Karmacharya PC, Koirala S, Tuladhar NR, Bryan LE, et al. (1991) Epidemiologic characteristics, predisposing factors, and etiologic diagnosis of corneal ulceration in Nepal. Am J Ophthalmol 111: 92-99.

6. Gopinathan U, Garg P, Fernandes M, Sharma S, Athmanathan S, et al. (2002) The epidemiological features and laboratory results of fungal keratitis: a 10-year review at a referral eye care center in South India. Cornea 21: 555-559.

7. Panda A, Sharma N, Das G, Kumar N, Satpathy G (1997) Mycotic keratitis in children: epidemiologic and microbiologic evaluation. Cornea 16: 295-299.

8. Tanure MA, Cohen EJ, Sudesh S, Rapuano CJ, Laibson PR (2000) Spectrum of fungal keratitis at Wills Eye Hospital, Philadelphia, Pennsylvania. Cornea 19: 307-312.

9. Basak SK, Basak S, Mohanta A, Bhowmick A (2005) Epidemiological and microbiological diagnosis of suppurative keratitis in Gangetic West Bengal, eastern India. Indian J Ophthalmol 53: 17-22.

10. Vemuganti GK, Garg P, Gopinathan U, Naduvilath TJ, John RK, et al. (2002) Evaluation of agent and host factors in progression of mycotic keratitis: A histologic and microbiologic study of 167 corneal buttons. Ophthalmology 109: 1538-1546.

11. Sun RL, Jones DB, Wilhelmus KR (2007) Clinical characteristics and outcome of Candida keratitis. Am J Ophthalmol 143: 1043-1045.

12. Khoo SH, Bond J, Denning DW (1994) Administering amphotericin B--a practical approach. J Antimicrob Chemother 33: 203-213.

13. Srinivasan M (2004) Fungal keratitis. Curr Opin Ophthalmol 15: 321-327.

14. Marangon FB, Miller D, Giaconi JA, Alfonso EC (2004) In vitro investigation of voriconazole susceptibility for keratitis and endophthalmitis fungal pathogens. Am J Ophthalmol 137: 820-825.

15. Rex JH, Pfaller MA (2002) Has antifungal susceptibility testing come of age? Clin Infect Dis 35: 982-989. 